\title{
Disrupting Reconsolidation of Conditioned Withdrawal Memories in the Basolateral Amygdala Reduces Suppression of Heroin Seeking in Rats
}

\author{
Kim G. C. Hellemans, Barry J. Everitt, and Jonathan L. C. Lee \\ Department of Experimental Psychology, University of Cambridge, Cambridge, CB2 3EB, United Kingdom
}

Recent data from our laboratory have demonstrated that appetitive drug memories undergo protein synthesis-dependent reconsolidation in the basolateral amygdala (BLA), an area important in the formation of emotional memories. We here investigated the importance of the BLA in the reconsolidation of opiate conditioned withdrawal memories. Rats with bilateral cannulas implanted in the BLA were trained to respond for heroin $(0.12 \mathrm{mg} / \mathrm{kg}$, i.v. $)$ under a seeking-taking schedule, which required responding on a seeking lever to gain the opportunity to self-administer heroin by a single response on a taking lever. After induction of opiate dependence with subcutaneously implanted, heroin-filled osmotic minipumps $\left(3 \mathrm{mg} \cdot \mathrm{kg}^{-1} \cdot \mathrm{d}^{-1}\right.$ heroin), rats received five consecutive pairings of a conditioned stimulus (CS) (tone, light, and odor compound) paired with naloxone $(0.10 \mathrm{mg} / \mathrm{kg}$, s.c.)-precipitated withdrawal. We replicated our previous findings that heroin seeking is suppressed in the presence of the withdrawal-associated CS. However, infusion of Zif268 antisense oligodeoxynucleotide into the BLA before reactivation of the CS-withdrawal association abolished this conditioned suppression in a reactivation-dependent manner. We also report that reconsolidation of CS-withdrawal memories upregulates Zif268 protein in the basolateral but not central nucleus of the amygdala and that Zif268 knockdown occurs selectively in the BLA. These results demonstrate that drug withdrawal memories undergo protein synthesis-dependent reconsolidation in the BLA and suggest a common mechanism for the reconsolidation of both appetitive and aversive drug memories.

Key words: amygdala; opiate; seeking-taking schedule; Zif268; self-administration; naloxone

\section{Introduction}

The reactivation of drug withdrawal memories by cues or contexts previously associated with withdrawal can motivate compulsive drug seeking in abstinent opiate addicts (Wikler, 1973; Childress et al., 1986). Wikler (1948) postulated that the reactivation of drug withdrawal memories occurred after repeated pavlovian pairing of stimuli associated with opiate withdrawal, leading to a state of "conditioned withdrawal." One prediction of this theory is that reexposure to stimuli previously paired with withdrawal should increase opiate seeking to relieve the negative affective state induced by conditioned withdrawal. Nonetheless, this has been difficult to demonstrate in experimental models, because stimuli paired with the experience of withdrawal do not increase (Wikler and Pescor, 1967; Miller et al., 1979) and may even suppress (Hellemans et al., 2006) drug seeking in opiatedependent animals.

The neural mechanisms underlying the formation and expres-

Received July 20, 2006; revised 0ct. 5, 2006; accepted 0ct. 27, 2006.

This research was supported by Medical Research Council (MRC) Programme Grant G9536855 (B.J.E.) and was conducted within the MRC/Wellcome Trust Behavioral and Clinical Neuroscience Institute. K.G.C.H. was supported by a Natural Sciences and Engineering Research Council postdoctoral fellowship.

Correspondence should be addressed to Kim G. C. Hellemans at her present address: Department of Cellular and Physiological Sciences, University of British Columbia, 2350 Health Sciences Mall, Vancouver, British Columbia, Canada V6T 1Z3. E-mail: khelle@interchange.ubc.ca.

DOI:10.1523/JNEUROSCI.3101-06.2006

Copyright $\odot 2006$ Society for Neuroscience ～0270-6474/06/2612694-06\$15.00/0 sion of conditioned opiate withdrawal involve the amygdala (Schulteis et al., 2000; Frenois et al., 2005). One way to assess whether amygdala-dependent conditioned stimulus (CS)-drug withdrawal associations are similar to other CS- unconditioned stimulus (US) memories is to investigate the propensity of CSwithdrawal memories to undergo reconsolidation in the basolateral amygdala (BLA). According to current reconsolidation theories, reactivation of a consolidated memory renders it once again vulnerable to amnestic treatment (Misanin et al., 1968), and the so-called reconsolidation of this old memory requires de novo protein synthesis (Nader et al., 2000; Nader, 2006). Studies from our laboratory have shown that appetitive drug memories undergo protein synthesis-dependent reconsolidation in the BLA. Intra-BLA infusions of antisense oligodeoxynucleotides (ASOs) targeting the immediate-early gene Zif268 before memory reactivation of a CS-cocaine or CS-fear association resulted in a significant reactivation-dependent reconsolidation deficit (Lee et al., 2005) and prevented cue-maintained cocaine seeking and cue-induced relapse (Lee et al., 2006). Expression of Zif268 is upregulated in the BLA after reexposure to CSs previously paired with both cocaine (Thomas et al., 2003) and footshock (Hall et al., 2001), and intra-BLA Zif268 ASO knocks down Zif268 protein in the BLA after reexposure to a CS paired with footshock (Lee et al., 2005). Thus, the synthesis of Zif268 in the BLA is required for the reconsolidation of both fear- and drugassociated memories. 
The purpose of the study was to investigate whether aversive drug memories (specifically, cues associated with opiate withdrawal) also undergo protein synthesis-dependent reconsolidation in the BLA. Rats were trained to respond for heroin using a well established seeking-taking task (Olmstead et al., 2000), and then withdrawal precipitated by low dose naloxone injection was paired with a discrete CS using the same conditioned suppression protocol used in our previous study (Hellemans et al., 2006). The involvement of the BLA in the reconsolidation of CS-elicited withdrawal memories was assessed by infusing Zif268 ASO directly into the BLA before memory reactivation, and conditioned suppression of heroin seeking was measured in a subsequent test session. To further assess the importance of Zif268 protein synthesis in the BLA on reconsolidation of CS-withdrawal memories, Zif268 upregulation and knockdown were measured in the BLA and central nucleus of the amygdala (CeN) using Western blotting analysis.

\section{Materials and Methods}

Self-administration training and dependence induction. Adult male Lister Hooded rats were surgically implanted with both a single indwelling intravenous catheter and bilateral BLA cannulas and, after recovery, were initially trained to respond for heroin $(0.12 \mathrm{mg} / \mathrm{kg}$ over $7.3 \mathrm{~s})$ under a fixed ratio (FR) 1 schedule of reinforcement in daily, $3 \mathrm{~h}$ sessions. After $5 \mathrm{~d}$, rats were then introduced to the multiple heterogeneous chain [tandem FR 1 (random interval $120 \mathrm{~s}$ ) FR 1] timeout schedule, which, for brevity, we refer to as a seeking-taking chain schedule. After stable responding, rats were implanted subcutaneously with osmotic minipumps (Charles River, Kent, UK) that infused heroin at a continuous rate (3 $\mathrm{mg} \cdot \mathrm{kg}^{-1} \cdot \mathrm{d}^{-1}$ ) for up to 4 weeks. A full description of the methods is available in the supplemental data (available at www.jneurosci.org as supplemental material).

Withdrawal conditioning. Conditioning of an intermittent tone, light, and odor compound stimulus with the naloxone-precipitated withdrawal state was performed over 5 consecutive days. For each session, rats were connected to the tether inside the chamber and then presented with the intermittent tone $(75 \mathrm{db}, 100 \mathrm{kHz})$, flashing light $(30 \mathrm{~W})$, and distinct odor (sandalwood essence; The Body Shop, Littlehampton, West Sussex, UK) for $2 \mathrm{~min}$. Rats were then removed from the chamber, injected with naloxone $(0.1 \mathrm{mg} / \mathrm{kg}$, s.c. $)$, and then immediately returned to the chamber for an additional $15 \mathrm{~min}$. Neither the seeking nor taking lever was present in the chamber at this time.

Experiment 1. To replicate previous findings (Hellemans et al., 2006) and ensure that animals could learn the CS-US relationship after BLA cannula implantation [cannulation of the BLA can sometimes have nonspecific effects on aversive learning and memory (Fendt, 2001)], animals were tested for conditioned suppression of heroin seeking in extinction (i.e., heroin was not available). During these sessions, animals were placed in the operant chamber for 2 min before receiving an injection of saline $(1 \mathrm{ml} / \mathrm{kg})$; they were then returned to the operant chamber for an additional $15 \mathrm{~min}$. During these sessions, the seeking lever was present in the chamber, but the taking lever was always retracted. Half of the rats in each group received the tone, light, and odor CS before and throughout the lever presentation, and half received no CS. Animals were then retrained on the seeking-taking chain schedule for two consecutive sessions, and then the presentation of the CS was reversed between animals (i.e., animals that did not receive the CS then received a test session with the CS). Sessions lasted $15 \mathrm{~min}$, and the total number of responses on the heroin-seeking lever was recorded.

Experiment 2. After the initial extinction testing, animals were reimplanted with osmostic minipumps, and they then received 5 additional consecutive days of withdrawal conditioning (i.e., naloxone-CS pairings). The following day, rats received intra-BLA infusions of either Zif268 ASOs $(n=8)$ or scrambled missense sequences (MSOs) $(n=9)$. Ninety minutes later, the CS-withdrawal association was reactivated in a single 2 min session, in which rats were reexposed continuously to the
CS. The next day, animals were tested for heroin seeking in the presence and absence of the CS in extinction sessions as described above.

Experiment 3. In a separate group of rats, reactivation-dependent disruption of reconsolidation was tested in animals that were not reactivated after Zif268 ASO infusion. Animals were trained, implanted with minipumps, subjected to the withdrawal conditioning procedure, and infused with Zif268 ASOs $(n=8)$ or MSOs $(n=9)$ as described above. However, groups did not receive a reactivation session but instead were returned to their home cage after infusions. The following day, animals were tested for CS suppression in extinction as described above.

Histological and Western blotting procedures. At the end of the experiment, rats from experiments 1 and 2 were perfused with paraformaldehyde, and their brains were extracted and cut using a cryostat to produce $60 \mu \mathrm{m}$ coronal sections. Slices were stained using cresyl violet, and accurate cannula placement was assessed using light microscopy. Results showed 17 rats with accurate placements.

Rats from experiment 3 were reconditioned for subsequent analysis of Zif268 protein levels after CS reexposure and Zif268 ASO infusion. Rats were split into four groups:)1) non-reactivated home cage control $(n=$ 4); (2) reactivated with no infusion $(n=4)$; (3) reactivated after Zif268 MSO infusion $(n=4)$; or (4) reactivated after Zif268 ASOs $(n=5)$. After CS withdrawal reactivation or non-reactivation, rats were killed by carbon dioxide inhalation. A full description of the Western blotting procedure is in the supplemental data (available at www.jneurosci.org as supplemental material).

Statistics. A full description of the statistical analyses can be found at in the supplemental data (available at www.jneurosci.org as supplemental material).

\section{Results}

All rats included in the behavioral analyses had cannulas located bilaterally in the BLA (Fig. 1).

Figure 2 shows that Zif268 ASOs infused into the BLA resulted in a significant, reactivation-dependent impairment in the CS suppression of heroin seeking (reactivation $X$ infusion interaction, $\left.F_{(2,45)}=7.87 ; p<0.05\right)$. Planned comparisons between pre-reactivation and post-reactivation conditions revealed a significant reactivation $\times$ infusion interaction $\left(F_{(1,34)}=9.09 ; p<\right.$ $0.05)$ and a significant main effect of infusion $\left(F_{(1,34)}=11.56 ; p<\right.$ $0.05)$. Post hoc analyses revealed that, whereas rats to be infused with ASOs or MSOs showed equivalent suppression of heroin seeking in the presence of the CS (pre-reactivation), infusion of Zif268 ASOs before reactivation with the CS paired previously with naloxone-precipitated withdrawal significantly impaired subsequent conditioned suppression (post-reactivation; $p<$ $0.05)$. Moreover, a single sample $t$ test revealed that the suppression ratio in ASO-infused animals in the post-reactivation condition was not significantly different from $0.5\left(t_{(7)}=-0.41 ; p=\right.$ 0.35 , one-tailed), further demonstrating that ASO-infused animals were not suppressed in the presence of the CS. Although MSO-infused rats were significantly more suppressed postreactivation compared with pre-reactivation $(p<0.05)$, this is likely attributable to the fact that animals in the postreactivation condition received an additional five CS-withdrawal pairings before infusion and testing. Importantly, planned comparisons between post-reactivation and nonreactivated groups revealed a significant reactivation $\times$ infusion interaction $\left(F_{(1,34)}=20.04 ; p<0.05\right)$; post hoc analyses indicated that this difference was attributable to the significant increase in suppression of heroin seeking in the nonreactivated ASO group compared with ASO-infused animals in the post-reactivation condition $(p<0.05)$. Also, rats that received ASO infusions in the non-reactivated control group did not differ from MSO-infused controls $(p>0.05)$. 


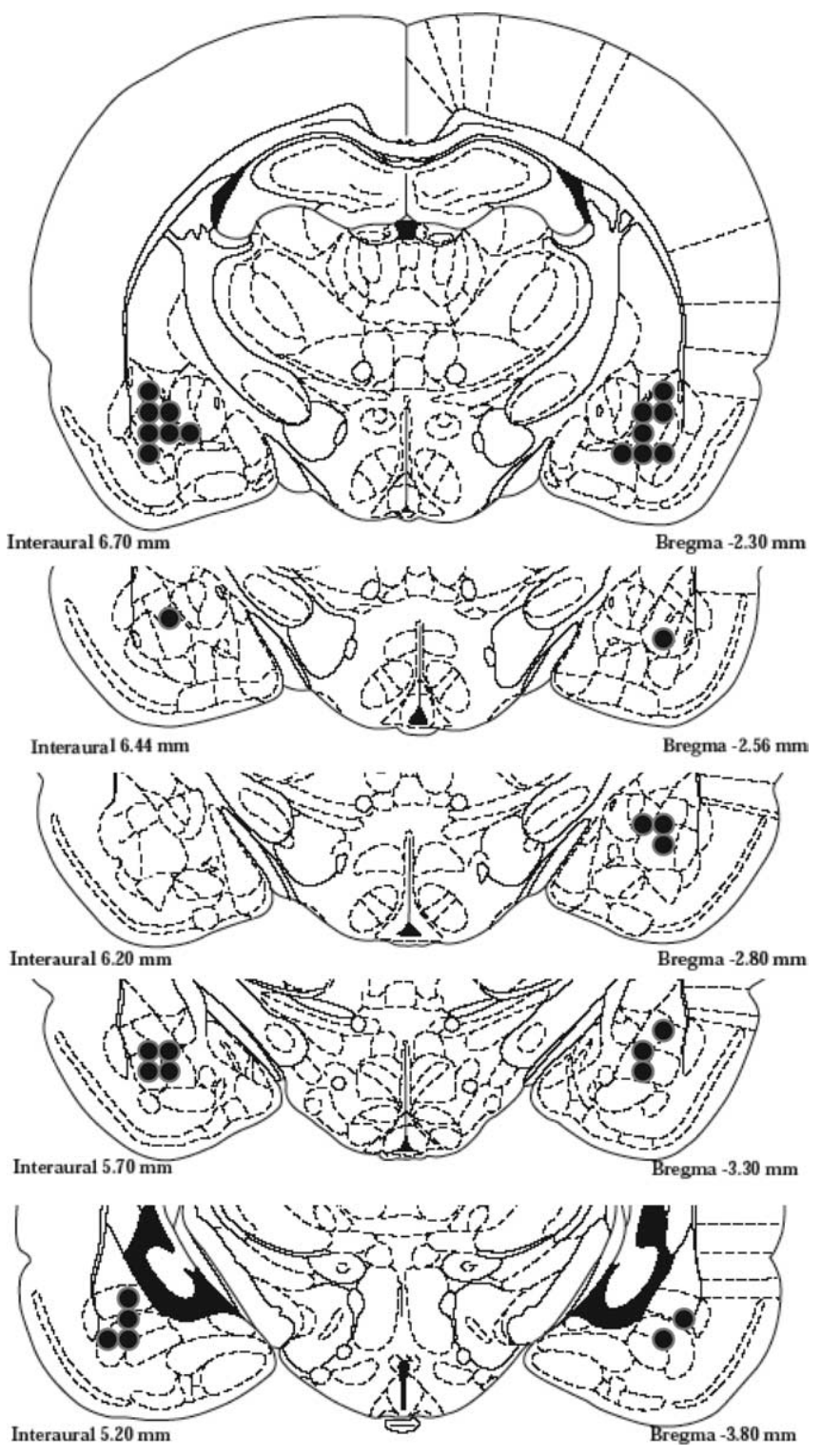

Figure 1. BLA cannulations. Schematic representation of the amygdala at five different rostrocaudal planes. Numbers represent the posterior coordinate from bregma (in millimeters). Injector tips for each cannulation are represented by filled circles.

Reexposure to the CS previously paired with naloxoneprecipitated withdrawal upregulated Zif268 protein specifically in the BLA (Fig. 3A). Microdissected BLA and CeN samples were separated on $7.5 \%$ Tris- $\mathrm{HCl}$ gels, and Zif268 protein levels were determined by Western blot analysis. Loading variation was corrected by normalizing the Zif268 amount according to the amount of $\beta$-actin in each sample (for additional details, see supplemental data, available at www.jneurosci.org as supplemental material). This analysis revealed a significant increase in Zif268 protein in the $\operatorname{BLA}\left(F_{(1,6)}=11.82 ; p<0.02\right)$ but not in the $\operatorname{CeN}\left(F_{(1,6)}=0.043 ; p>0.84\right)$ for rats reexposed to the CS compared with non-reexposed controls. However, infusion of Zif268 ASOs into the BLA before CS reexposure attenuated this increase in Zif268 protein (Fig. 3B). Zif268 ASOs resulted in a $20 \%$ knockdown in the $\operatorname{BLA}\left(F_{(1,7)}=10.82\right.$; $p<0.02)$ but had no effect on Zif268 protein levels in the CeN $\left(F_{(1,6)}=0.161 ; p>0.70\right)$.

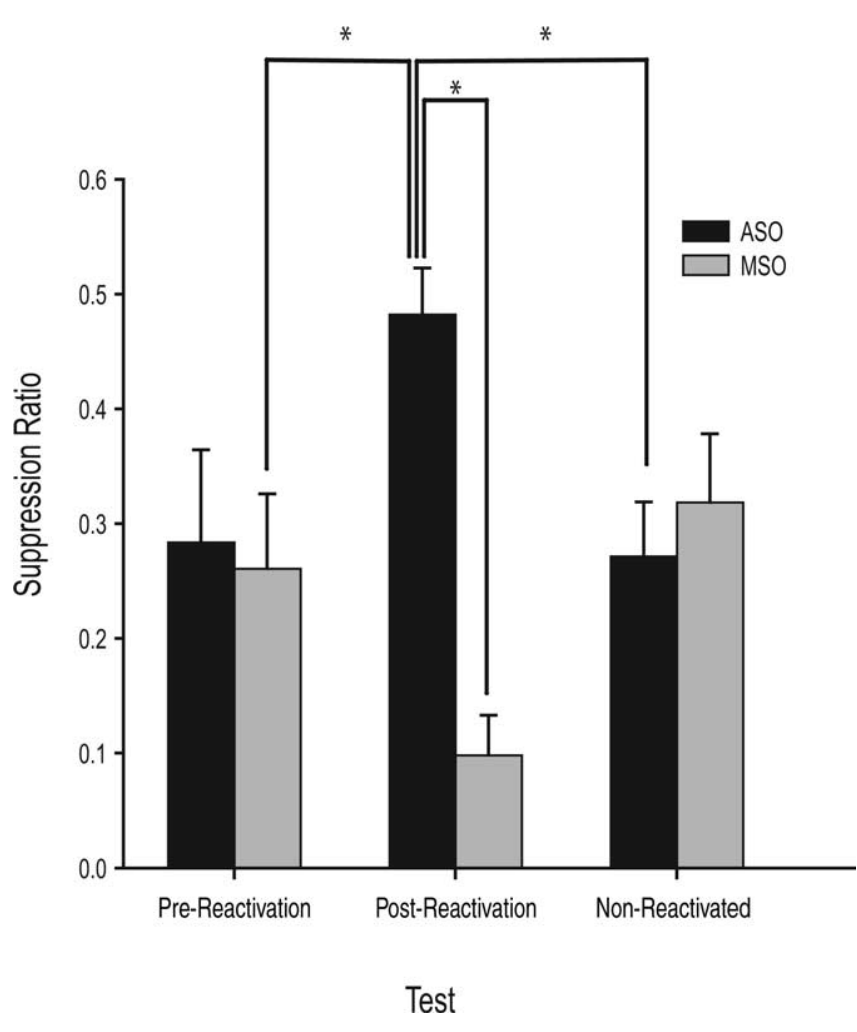

Figure 2. Intra-BLA Zif268 ASOs impairs conditioned suppression to a CS previously associated with naloxone-precipitated withdrawal. Bars represent the mean \pm SEM suppression ratio of heroin-seeking responses [CS On/(CS On + CS Off); suppression ratio of 0.5 represents no suppression and $<0.5$ indicates $(S$-induced suppression] before reactivation, after reactivation, and in a non-reactivated control group. Infusion of Zif268 ASOs impaired subsequent conditioned suppression of heroin seeking relative to both control MSO infusions and baseline pre-reactivation levels. Reconsolidation of the conditioned withdrawal memories is reactivation dependent: animals that did not receive reactivation with the $\mathrm{CS}$, but did receive ASO infusions, showed no difference in suppression ratios compared with MSO-infused controls. ${ }^{*} p<0.05$

\section{Discussion}

The results of the present experiments demonstrate that aversive drug memories undergo protein synthesis-dependent reconsolidation in the amygdala in a manner similar to appetitive drug memories. Specifically, we show that intra-BLA Zif268 ASO infusions before a brief period of memory reactivation through reexposure to a CS associated with opiate withdrawal abolishes conditioned suppression of heroin seeking measured in a subsequent test session. This finding was critically dependent on memory reactivation with the withdrawal-associated CS, because nonreactivated rats infused with Zif268 ASO showed no impairment. Moreover, Western blotting analysis showed that Zif268 is upregulated in the BLA, but not $\mathrm{CeN}$, after memory reactivation through reexposure to the withdrawal-paired CS, and intra-BLA Zif268 ASO infusion before memory reactivation significantly attenuated this increase. Therefore, the behavioral effects of Zif268 ASO infusion are most likely mediated by the knockdown of Zif268 protein in the BLA.

Initially, all rats in the pre-reactivated condition showed significant suppression of heroin seeking in the presence of the CS associated previously with naloxone-precipitated withdrawal. These data support both our previous and other findings that stimuli paired with the aversive experience of opiate withdrawal suppress appetitive behavior in opiate-dependent rats (Goldberg and Schuster, 1967; Goldberg and Schuster, 1970; Gellert and 
A.
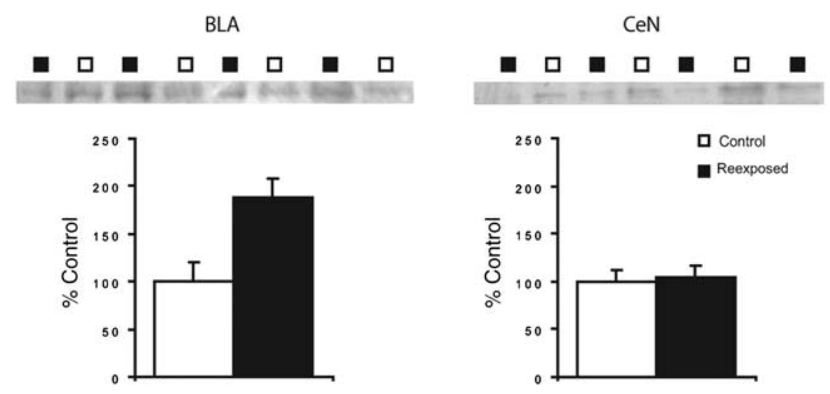

B.
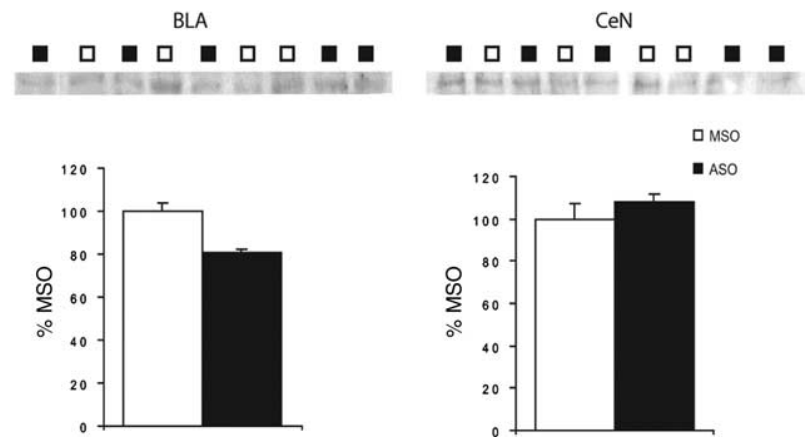

Figure 3. A, Zif268 protein levels after reexposure to a conditioned withdrawal stimulus. Zif268 protein was upregulated $2 \mathrm{~h}$ after stimulus reexposure in the BLA but not in the $\mathrm{CeN} ; p<$ 0.05 . Data are presented as mean \pm SEM. $\boldsymbol{B}$, Zif268 protein levels after reexposure to a conditioned withdrawal stimulus and Zif268 ASO infusion. Zif268 ASOs resulted in a significant decrease in Zif268 expression compared with rats infused with Zif268 MSOs, $2 \mathrm{~h}$ after stimulus reexposure in the BLA but not in the CeN; $p<0.05$. Data are presented as mean \pm SEM.

Sparber, 1977; Koob et al., 1989; Hellemans et al., 2006). Importantly, our data also demonstrate that BLA-cannulated rats are capable of learning the CS-withdrawal association and verify that rats that were later infused with ASOs showed no preexisting impairment in instrumental learning. In contrast, in postreactivation test sessions, rats that were infused with Zif268 ASOs into the BLA showed impaired suppression of heroin seeking compared with both MSO-infused controls and their own prereactivation levels. Indeed, Zif268 ASO-infused rats did not show any suppression of heroin seeking when the withdrawal-associated CS was presented. Thus, blocking reconsolidation of the CS-withdrawal memory through infusion of Zif268 ASO at memory reactivation abolishes the ability of the CS to suppress heroin seeking in the future. These data greatly extend our previous observations that intra-BLA Zif268 ASO disrupts the reconsolidation of appetitive drug-associated memories for drugpaired stimuli (Lee et al., 2005, 2006), by showing that aversive drug memories are also susceptible to disruption in this manner. Although MSO-infused animals in the post-reactivation session were significantly more suppressed than in the prereactivation test, this is likely attributable to the 5 additional consecutive days of withdrawal conditioning (i.e., naloxone-CS pairings) these animals received after the initial test session. That ASO-infused animals showed no suppression during this session despite the additional CS-withdrawal pairings is additional evidence of the strength of the amnesic effect after Zif268 knockdown in the BLA.

A critical criterion for the demonstration of a reconsolidation impairment is that the amnesic effect is observed only after memory reactivation and not if this reactivation session is omitted (Lewis, 1979; Dudai, 2004). In our study, animals that did not experience reactivation of the CS-withdrawal memory but were infused with Zif268 ASOs before testing showed the same degree of conditioned suppression of heroin seeking as that seen in the MSO-infused controls. Thus, our data confirm that the effects of Zif268 ASO on the impairment of appetitive and aversive memory reconsolidation are reactivation dependent (Lee et al., 2005, 2006; Debiec et al., 2006). Some studies have demonstrated a recovery from amnesia after reconsolidation blockade with repeated testing (Lattal and Abel, 2004; Power et al. 2006), whereas others have found no evidence of recovery (Duvarci and Nader, 2004; Lee et al., 2004). Although the current study did not test for persistent impairments in heroin seeking, previous data from our laboratory using Zif268 ASOs to explore both appetitive and aversive memory reconsolidation deficits have never observed any indication of a recovery, suggesting a persistent amnesia after this manipulation (Lee et al., 2004, 2005, 2006).

Regulation of Zif268, an inducible transcription factor, appears to be critical for many forms of associative learning (Davis et al., 2003) and may be a common fundamental requirement for the reconsolidation of aversive and appetitive memories (Lee et al., 2004, 2005). Our data are in accordance with this finding, because we found that Zif268 was upregulated in the BLA after reexposure to an opiate withdrawal-paired cue. Our results also suggest a selective involvement of the BLA in regulating reconsolidation of CS-withdrawal memories, because Zif268 was not upregulated in the CeN after reactivation with the CS, and Zif268 ASO infusion reduced expression selectively in the BLA. This latter finding extends the data in previous reports not only by confirming that Zif268 ASO attenuates Zif268 upregulation in the BLA (Lee et al., 2005) and whole amygdala (Malkani et al., 2004) but also providing the novel finding that the effect is selective to the BLA, because there is no reduction of Zif268 protein in the CeN. The amygdalar nuclei form part of an interconnected circuit that has been suggested to underlie the aversive aspects of opiate withdrawal (Frenois et al., 2002, 2005). A wealth of evidence suggests that, whereas the $\mathrm{CeN}$ is activated during primary withdrawal, the BLA appears to be selectively involved in mediating the formation and expression of conditioned opiate withdrawal. Lesions of the BLA abolish conditioned, but not unconditioned, suppression of instrumental responding for food in opiate-dependent rats (Schulteis et al., 2000), and increased c-fos expression occurs selectively in the BLA after reexposure to an environment previously paired with naloxone-precipitated withdrawal (Frenois et al., 2005). Thus, we add to this growing body of evidence by using reversible protein knockdown to show that the BLA is functionally involved in conditioned opiate withdrawal and extend these findings to show that the reconsolidation of CS-withdrawal memories occurs selectively within the BLA.

Although this experiment is unique in showing that aversive drug withdrawal-associated memories undergo protein synthesis-dependent reconsolidation in the BLA, an important extension of this finding would be to explore whether the reconsolidation of incentive learning related to drug-paired stimuli can also be disrupted. Thus, we have shown that a CS previously paired with naloxone-precipitated withdrawal initially suppressed heroin seeking in opiate-dependent rats but enhanced heroin seeking when this CS was later paired with heroin taking (Hellemans et al., 2006). This latter finding supports previous reports that drug self-administration in the presence of a withdrawal-paired CS increases drug seeking 
during the conditioned withdrawal state (Goldberg et al., 1969; Kenny et al., 2006). However, these results may be explained by the operation of incentive-learning processes underlying drug addiction whereby drug seeking in withdrawal may be rendered compulsive when the drug is previously experienced in a withdrawn state, providing the opportunity for a subject to learn about its increased value in that state (Dickinson and Balleine, 1994, 2002; Hutcheson et al., 2001). If we seek to understand how drug-associated memories control and lead to compulsive drug-seeking behavior, an important test would be to explore whether the CS now associated with the increased incentive value of heroin also undergoes protein synthesis-dependent reconsolidation in the BLA. There is good evidence to suggest that the BLA is involved in incentive learning (Everitt et al., 1999; Balleine et al., 2003) and consolidation of memory for changes in the incentive value of rewards (Salinas et al., 1993). Moreover, reconsolidation of incentive memory for different food outcomes is blocked by intra-BLA infusions of anisomycin (Wang et al., 2005), suggesting that incentive learning for natural rewards undergoes protein synthesis-dependent reconsolidation in the BLA. Future studies that examine this phenomenon with drug-related stimuli could provide important information as to how incentive learning for appetitive drug-associated stimuli controls compulsive drug-seeking behavior.

In summary, this study provides novel and important information on the formation of CS-withdrawal associations, suggesting that aversive drug memories undergo protein synthesis-dependent reconsolidation in the BLA in a reactivation-dependent manner. The BLA is part of an integrated circuit of neural structures implicated in the formation and storage of CS-US associations (Everitt et al., 1999, 2003) and in the processing of emotional events in relation to environmental stimuli that guide motivated behavior (Cardinal et al., 2002). Our data add to the growing body of evidence that the BLA is also involved in the reconsolidation of fear, drug, and incentive memories (Nader et al., 2000; Lee et al., 2005, 2006; Wang et al., 2005) and extend these findings to include aversive drug memories retrieved by presenting opiate withdrawal-paired CSs. Together, these studies provide critical information on how reexposure to pavlovian cues associated with both drug intake and drug withdrawal may influence instrumental behavior to render drug-seeking compulsive in abstinent addicts.

\section{References}

Balleine BW, Killcross AS, Dickinson A (2003) The effects of lesions of the basolateral amygdala on instrumental conditioning. J Neurosci 23:666-675.

Cardinal RN, Parkinson JA, Hall J, Everitt BJ (2002) Emotion and motivation: the role of the amygdala, ventral striatum, and the frontal cortex. Neurosci Biobehav Rev 26:321-352.

Childress AR, McLellan AT, O'Brien CP (1986) Abstinent opiate abusers exhibit conditioned craving, conditioned withdrawal and reductions in both through extinction. Br J Addict 81:655-660.

Davis S, Bozon B, Laroche S (2003) How necessary is the activation of the immediate early gene zif 268 in synaptic plasticity and learning. Behav Brain Res 142:17-30.

Debiec J, Doyère V, Nader K, LeDoux JE (2006) Directly reactivated, but not indirectly reactivated, memories undergo reconsolidation in the amygdala. Proc Natl Acad Sci USA 103:3428-3433.

Dickinson A, Balleine BW (1994) Motivational control of goal-directed action. Anim Learn Behav 22:1-18.

Dickinson A, Balleine BW (2002) The role of learning in the operation of motivation systems. In: Steven's handbook of experimental psychology: learning, motivation and emotion (Pashler H, Gallistel CR, eds), New York: Wiley.

Dudai Y (2004) The neurobiology of consolidations, or, how stable is the engram? Annu Rev Psychol 55:51-86.

Duvarci S, Nader K (2004) Characterization of fear memory reconsolidation. J Neurosci 24:9269-9275.

Everitt BJ, Parkinson JA, Olmstead MC, Arroyo M, Robledo P, Robbins TW (1999) Associative processes in addiction and reward: the role of amygdala-ventral striatal subsystems. Ann NY Acad Sci 877:412-438.

Everitt BJ, Cardinal RN, Parkinson JA, Robbins TW (2003) Appetitive behavior: impact of amygdala-dependent mechanisms of emotional learning. Ann NY Acad Sci 985:233-250.

Fendt M (2001) Injections of the NMDA receptor antagonist aminophosphonopentanoic acid into the lateral nucleus of the amygdala block the expression of fear-potentiated startle and freezing. J Neurosci 21:4111-4115.

Frenois F, Cador M, Caille S, Stinus L, Le Moine C (2002) Neural correlates of the motivational and somatic components of naloxone-precipitated morphine withdrawal. Eur J Neurosci 16:1377-1389.

Frenois F, Stinus L, Di Blasi F, Cador M, Le Moine C (2005) A specific limbic circuit underlies opiate withdrawal memories. J Neurosci 25:1366-1374.

Gellert VF, Sparber SB (1977) A comparison of the effects of naloxone upon body weight loss and suppression of fixed-ratio operant behavior in morphine-dependent rats. J Pharmacol Exp Ther 201:44-54.

Goldberg SR, Schuster CR (1967) Conditioned suppression by a stimulus associated with nalorphine in morphine-dependent monkeys. J Exp Anal Behav 10:235-242.

Goldberg SR, Schuster CR (1970) Conditioned nalorphine-induced abstinence changes: persistence in post morphine-dependent monkeys. J Exp Anal Behav 14:33-46.

Goldberg SR, Woods JH, Schuster CR (1969) Morphine: conditioned increases in self-administration in rhesus monkeys. Science 166:1306-1307.

Hall J, Thomas KL, Everitt BJ (2001) Cellular imaging of zif268 expression in the hippocampus and amygdala during contextual and cued fear memory retrieval: selective activation of hippocampal CA1 neurons during the recall of contextual memories. J Neurosci 21:2186-2193.

Hellemans KGC, Dickinson A, Everitt BJ (2006) Motivational control of heroin seeking by conditioned stimuli associated with withdrawal and heroin taking by rats. Behav Neurosci 120:103-114.

Hutcheson DM, Everitt BJ, Robbins TW, Dickinson A (2001) The role of withdrawal in heroin addiction: enhances reward or promotes avoidance? Nat Neurosci 4:943-947.

Kenny PJ, Chen SA, Kitamura O, Markou A, Koob GF (2006) Conditioned withdrawal drives heroin consumption and decreases reward sensitivity. J Neurosci 26:5894-5900.

Koob GF, Wall TL, Bloom FE (1989) Nucleus accumbens as a substrate for the aversive stimulus effects of opiate withdrawal. Psychopharmacology (Berl) 98:530-534.

Lattal KN, Abel T (2004) Behavioral impairments caused by injections of the protein synthesis inhibitor anisomycin after contextual retrieval reverse with time. Proc Natl Acad Sci USA 30:4667-4672.

Lee JLC, Everitt BJ, Thomas KL (2004) Independent cellular processes for hippocampal memory consolidation and reconsolidation. Science 304:839-843.

Lee JLC, Di Ciano P, Thomas KL, Everitt BJ (2005) Disrupting reconsolidation of drug memories reduces cocaine-seeking behavior. Neuron 47:795-801.

Lee JLC, Milton AL, Everitt BJ (2006) Cue-induced cocaine seeking and relapse are reduced by disruption of drug memory reconsolidation. J Neurosci 26:5881-5887.

Lewis DJ (1979) Psychobiology of active and inactive memory. Psychol Bull 86: $1054-1083$.

Malkani S, Wallace KJ, Donley MP, Rosen JB (2004) An egr-1 (zif268) Antisense oligodeoxynucleotide infused into the amygdala disrupts fear conditioning. Learn Mem 11:617-624.

Miller DB, Dougherty JA, Wikler A (1979) Interoceptive conditioning through repeated suppression of morphine-abstinence. II. Relapse testing. Pavlov J Biol Sci 14:170-176.

Misanin JR, Miller RR, Lewis DJ (1968) Retrograde amnesia produced by 
electroconvulsive shock after reactivation of a consolidated memory trace. Science 160:554-555.

Nader K (2006) Memory traces unbound. Trends Neurosci 26:65-72.

Nader K, Schafe GE, LeDoux JE (2000) Fear memories require protein synthesis in the amygdala for reconsolidation after retrieval. Nature 406:722-726.

Olmstead MC, Parkinson JA, Miles FJ, Everitt BJ, Dickinson A (2000) Cocaine-seeking by rats: regulation, reinforcement and activation. Psychopharmacology 152:123-131.

Power AE, Berlau DJ, McGaugh JL, Steward O (2006) Anisomycin infused into the hippocampus fails to block "reconsolidation" but impairs extinction: the role of re-exposure duration. Learn Mem 13:27-34.

Salinas JA, Packard MG, McGaugh JL (1993) Amygdala modulates memory for changes in reward magnitude: reversible post-training inactivation with lidocaine attenuates the response to a reduction in reward. Behav Brain Res 59:153-159.
Schulteis G, Ahmed SH, Morse AC, Koob GF, Everitt BJ (2000) Conditioning and opiate withdrawal. Nature 405:1013-1014.

Thomas KL, Arroyo M, Everitt BJ (2003) Induction of the learning and plasticity-associated gene Zif268 following exposure to a discrete cocaineassociated stimulus. Eur J Neurosci 17:1964-1972.

Wang S-H, Ostlund SB, Nader K, Balleine BW (2005) Consolidation and reconsolidation of incentive learning in the amygdala. J Neurosci 25:830-835.

Wikler A (1948) Recent progress in research on the neurophysiological basis of morphine addiction. Am J Psychiatry 105:329-338.

Wikler A (1973) Dynamics of drug dependence, implication of a conditioning theory for research and treatment. Arch Gen Psychiatry 28:611-616.

Wikler A, Pescor FT (1967) Classical conditioning of a morphine abstinence phenomenon, reinforcement of opioid-drinking behavior and "relapse" in morphine-addicted rats. Psychopharmacologia 10:255-284. 